\title{
Los conflictos de intereses en el marco jurídico mexicano: su indefinición normativa
}

\section{Conflict of interest in mexican law: its normative non-definition}

\author{
Jessica Eliane Padilla Ramírez ${ }^{[*]}$
}

\begin{abstract}
Resumen: en la legislación mexicana, la actuación de los servidores públicos bajo conflicto de interés se encuentra regulada como una falta administrativa grave, cuyas consecuencias pueden ir desde la suspensión temporal del cargo hasta la inhabilitación; sin embargo, se trata de un tipo administrativo de difícil determinación y sanción, como resultado de dos situaciones concretas: 1) es una práctica reiterada, normalizada y aceptada; y 2) la normatividad mexicana es imprecisa en lo que se refiere a esta conducta. Por lo tanto, el presente documento analiza los conflictos de intereses desde una perspectiva jurídica y, a partir de un enfoque práctico, propone elementos que faciliten su comprensión e identificación.
\end{abstract}

Palabras claves: conflictos de intereses, imparcialidad, corrupción, falta administrativa, servidores públicos.

Abstract: in mexican law, the conduct of public servants with conflict of interest is regulated as a serious administrative offense, the consequences of which can range from temporary suspension of office to disqualification; however, it is an administrative type of difficult determination and sanction, as a result of two specific situations: 1) it is a reiterated, standardized and accepted practice; and 2) mexican regulations are imprecise regarding this conduct. Therefore, this document analyzes conflicts of interest from a legal perspective and, from a practical approach, proposes elements that facilitate their understanding and identification.

Key words: conflict of interest, impartiality, corruption, administrative fault, public servants.

[*] Candidata a Maestra en Administración Pública por el Instituto Nacional de Administración Pública (INAP), México, Licenciada en Derecho y servidora pública. Contacto: jess.padillar@gmail.com 


\section{INTRODUCCIÓN}

Desde mis funciones como servidora pública, he tenido la oportunidad de intercambiar experiencias con funcionarios de los distintos niveles de la Administración Pública, resultando que la percepción que ellos mismos tienen sobre la existencia de conflictos de intereses no es muy diferente a la percepción de la ciudadanía y la sociedad civil, en el sentido de que, tanto las personas que laboramos en el servicio público, como quienes se encuentran fuera de este, reconocemos la tendencia a la priorización de los intereses privados sobre el interés público y, sin embargo, el común denominador es percibirlo como una situación normal.

Adicionalmente, gran parte de la problemática deriva de la consideración de que los conflictos de intereses constituyen una conducta indebida, cuando la realidad es que se trata de situaciones completamente normales en el día a día de servidores públicos; lo importante, entonces, es comprender la diferencia entre éstos y la conducta que configura la falta administrativa denominada «actuación bajo conflicto de interés».

La actuación bajo conflicto de interés se encuentra regulada en la Ley General de Responsabilidades Administrativas como una falta administrativa grave, cuyas consecuencias pueden ir desde la suspensión temporal del cargo hasta la inhabilitación; no obstante, se trata de un tipo administrativo de difícil determinación y más difícil de sancionar, como resultado de dos situaciones concretas:

1. Es una práctica reiterada, normalizada y aceptada, tanto para el sector público como para la sociedad.

2. La normatividad mexicana tiene imprecisiones y vacíos en lo que se refiere a esta conducta; además de que, a la fecha, no se cuenta con interpretaciones judiciales en la materia.

Lo anterior tiene como consecuencia la poca tipificación de conductas graves que afectan la imparcialidad en las determinaciones de los servidores públicos, el uso eficiente de los recursos, el desempeño objetivo de la Administración Pública y un largo etcétera; entonces surgen dos interrogantes principales: ¿Basta con reformar la normatividad para que la actuación bajo conflicto de interés sea sancionada efectivamente? Y, una vez hecho esto, ¿la determinación de las conductas que dan lugar a la actuación bajo conflicto de interés tendrá como efecto la erradicación de dicha práctica?

\section{LOS CONFLICTOS DE INTERESES: ANÁLI- SIS DE SU DEFINICIÓN}

Cuando hablamos de conflictos de intereses, nos referimos a situaciones que crean dilemas éticos en el desempeño del deber profesional, tanto en el sector público como en el privado, ya que cualquier persona puede encontrarse en un escenario en que se contrapongan sus intereses propios con los que corresponden a su actividad laboral; no obstante, para efectos del presente documento, nos enfocaremos en los que se refieren a los servidores públicos.

Es importante destacar que, todos los intereses que tiene o puede tener el servidor público como persona, son completamente legítimos; sin embargo, cuando se desempeña en un empleo, cargo o comisión, la mera existencia de dichos intereses cuestiona la imparcialidad y objetividad de su discernimiento profesional, así como la confianza pública; principios clave para el funcionamiento democrático (Parlamento de Cataluña, 2016, p. 28). Por este motivo, la presencia de conflictos de intereses representa un problema de suma relevancia para cualquier Estado de Derecho.

Ahora bien, es de observarse que no hay un concepto definitivo, ni a nivel nacional ni internacional, lo que da lugar a entenderlo en más de un sentido y, comúnmente, emplearlo para hacer referencia a distintos actos de corrupción que se le parecen, generando una situación de 
incertidumbre y confusión, tanto en la sociedad como en los diferentes órdenes de gobierno.

En la normatividad mexicana, el concepto jurídico vigente es el que se encuentra en la Ley General de Responsabilidades Administrativas: «La posible afectación del desempeño imparcial y objetivo de las funciones de los Servidores Públicos en razón de intereses personales, familiares o de negocios» (Artículo 3, fracción $\mathrm{VI}$ ).

En el mismo sentido el Acuerdo que tiene por objeto emitir el Código de Ética de los servidores públicos del Gobierno Federal, las Reglas de Integridad para el ejercicio de la función pública, y los Lineamientos generales para propiciar la integridad de los servidores públicos y para implementar acciones permanentes que favorezcan su comportamiento ético, a través de los Comités de Ética y de Prevención de Conflictos de Interés, reformado el 22 de agosto de 2017, que el conflicto de intereses es «la situación que se presenta cuando los intereses personales, familiares o de negocios del servidor público puedan afectar el desempeño independiente o imparcial de sus empleos, cargos, comisiones o funciones» (Numeral tercero, inciso f).

Bajo el razonamiento que siguen las anteriores definiciones, se destacan como elementos comunes la presencia de intereses personales, familiares o de negocios y, la afectación del desempeño imparcial de las funciones de los servidores públicos.

Por otro lado, resulta importante referir una definición que es ampliamente aceptada, tanto a nivel nacional como internacional; si bien ésta no resulta vinculante para el país mexicano, es de suma utilidad en la atención y prevención de los conflictos de intereses. En 2003, la Organización para la Cooperación y
Desarrollo Económicos (OCDE), en colaboración con países de las regiones Sureste de Europa, Asia-Pacifico y Latinoamérica, desarrolló un toolkit ${ }^{[1]}$ que propone soluciones prácticas para gestionar los conflictos de intereses en el sector público.

Dicho organismo formula también una clasificación de conflictos de intereses en tres tipos:

- Conflicto de interés real: Conflicto entre el deber público y los intereses privados del servidor público, en el que tiene intereses personales que pueden influir indebidamente en el desempeño de sus deberes y responsabilidades oficiales (OCDE, 2005, p. 13).

- Conflicto de interés aparente: Apariencia de que los intereses privados de un servidor público influyen indebidamente en el desempeño de sus deberes y responsabilidades oficiales, aunque no sea el caso (OCDE, 2005, p. 24).

- Conflicto de interés potencial: El servidor público tiene intereses privados susceptibles de provocar que el futuro se incurra en un conflicto de intereses real (OCDE, 2005, p. 25).

De las definiciones señaladas, cabe hacer ciertas precisiones: en el marco jurídico mexicano, el tipo de conflicto de interés que se encuentra regulado y que, como consecuencia deriva en una falta administrativa, es el conflicto de interés real, ello toda vez que es el único supuesto en que el servidor público se halla efectivamente ante una situación que afecta su objetividad e imparcialidad y, dependiendo el caso particular, resultará en la configuración de una falta administrativa grave.

No obstante, la clasificación presentada por la OCDE es relevante desde una perspectiva

[1] Este documento es un kit de herramientas que tiene por objetivo permitir a las personas servidoras públicas el reconocimiento de situaciones de conflictos de intereses y ayudarles a garantizar que la integridad y reputación no se vean comprometidas; así mismo, es útil para el desarrollo, adaptación y aplicación en cada contexto administrativo particular. 
de identificación y prevención, en razón de las consecuencias que generan a la Administración Pública. El conflicto de intereses aparente se encuentra presente en la mayoría de los casos en que se presume la existencia de un conflicto de interés real, puede ir desde meras aseveraciones hasta casos en los que existe alguna otra falta administrativa asociada a la corrupción y que se confunde en el conflicto de intereses; ello dependerá de un elemento indispensable que será analizado más adelante: el discernimiento.

Con independencia de ello, su mera presencia traerá como resultado la afectación de la imagen del servidor público y, consecuentemente, de la percepción sobre los índices de conductas asociadas a la corrupción.

Por su parte, el conflicto de intereses potencial requiere atención en razón de la susceptibilidad de configurarse en un conflicto de intereses real y, en su caso, la comisión de la falta administrativa correspondiente.

Ahora bien, es indispensable notar que los conflictos de intereses no implican de facto la configuración de una falta administrativa, hay que distinguir entre dos momentos: el conflicto de intereses en sí mismo y la actuación bajo conflicto de interés.

En términos de la Ley General de Responsabilidades Administrativas, se considera que:

Incurre en actuación bajo Conflicto de Interés el servidor público que intervenga por motivo de su empleo, cargo o comisión en cualquier forma, en la atención, tramitación o resolución de asuntos en los que tenga Conflicto de Interés o impedimento legal. (Artículo 58, primer párrafo).
Bajo este orden de ideas, en la siguiente tabla se muestra que, mientras el conflicto de interés es llanamente una situación causada por la presencia de un interés del servidor público y refleja un riesgo, la actuación bajo conflicto de interés implica una acción en que el servidor busca beneficiarse a sí mismo o a alguna de las personas que conforman su círculo de intereses ${ }^{[2]}$, constituyendo además una falta administrativa grave.

\section{Tabla 1}

Conflicto de Interés vs Actuación bajo Conflicto de Interés

\begin{tabular}{|l|l|}
\hline \multicolumn{1}{|c|}{ Conflicto de Interés } & \multicolumn{1}{|c|}{$\begin{array}{c}\text { Actuación bajo } \\
\text { Conflicto de Interés }\end{array}$} \\
\hline $\begin{array}{l}\text { Artículo 3, fracción VI de } \\
\text { la LGRA. }\end{array}$ & Artículo 58 de la LGRA. \\
\hline Es una situación & Es una acción. \\
\hline $\begin{array}{l}\text { Se produce por la existen- } \\
\text { cia de un interés personal, } \\
\text { familiar o de negocios. }\end{array}$ & $\begin{array}{l}\text { Se produce porque el servi- } \\
\text { ficio para sí mismo o para }\end{array}$ \\
\hline $\begin{array}{l}\text { Tiene como consculo de intereses. } \\
\text { cia el riesgo de actualizar } \\
\text { una falta administrativa. }\end{array}$ & $\begin{array}{l}\text { Es una falta administrativa } \\
\text { grave. }\end{array}$ \\
\hline
\end{tabular}

Fuente: Elaboración propia.

Es sustancial aclarar que, con independencia de que la parcialidad del servidor público se traduzca en el beneficio o perjuicio de un tercero, es posible considerar que ello configura un conflicto de interés, en razón de que, en ambos casos, la decisión del involucrado se ve nublada por un vínculo preexistente, actuando, en consecuencia, en sentido contrario a los principios de imparcialidad y objetividad.

[2] Concepto que la Secretaría de la Función Pública emplea para referirse a los vínculos susceptibles de dar lugar a la configuración de un conflicto de intereses, identificando el matrimonio, concubinato, parentesco consanguíneo, parentesco por afinidad, parentesco civil, terceros con los que se tienen relaciones profesionales, laborales o de negocios y socios o sociedades de las que todos los anteriores formen parte. Recuperado de http://funcionpublica.gob.mx/web/UnidadDeEtica/Conflictometro/ App_Conflictometro.pdf 


\subsection{Elementos}

Como se ha señalado en los párrafos anteriores, los conflictos de intereses son fenómenos complejos, de difícil determinación en la mayoría de los casos, por ello resulta necesario analizar los elementos que los integran a efecto de dilucidar si se configura o no la falta administrativa.

La principal dificultad a que nos enfrentamos al momento de realizar un análisis exhaustivo en la materia es el vacío existente en la normatividad y la falta de criterios judiciales, así como la escasa doctrina; por este motivo, a continuación se presenta una serie de aspectos que, en conjunto, configuran los conflictos de intereses:

\section{a) Sujeto involucrado}

En términos de la Ley General de Responsabilidades Administrativas, para que se configure un Conflicto de Intereses es necesario que, el sujeto involucrado que antepone sus intereses privados a los correspondientes al empleo, cargo o comisión que desempeña, sea un servidor público.

Entendiendo que se consideran servidores públicos los representantes de elección popular, los miembros del Poder Judicial de la Federación y, en general toda persona que desempeñe un empleo, cargo o comisión de cualquier naturaleza en el Congreso de la Unión o en la Administración Pública Federal, así como los servidores públicos de los Organismos Constitucionales Autónomos (Constitución Política de los Estados Unidos Mexicanos, 2020, Artículo 108).

Al respecto, cabe precisar que, según la Tesis Aislada de la Segunda Sala de la Suprema Corte de Justicia de la Nación, el artículo 108 Constitucional es enunciativo, no limitativo, en razón de la naturaleza del servicio a la sociedad, por lo que es posible incluir en la categoría de «servidores públicos» a todas las personas que sirven «al Estado o Federación, al gobierno y a la nación, al interés público o a la sociedad», sin importar la clase de empleo que desempeñen, ni el nivel de la función o la institución en donde laboren (Tesis Aislada 2 ${ }^{\mathrm{a}}$. XCIII/2006, N. ${ }^{\circ}$ 173672).

Bajo esta tesitura y conforme a lo dispuesto por el artículo 58 de la Ley General de Responsabilidades Administrativas, resulta interesante cuestionarse si las personas que prestan sus servicios en las dependencias o instituciones, mediante contratos civiles o que están sujetas al pago de honorarios, son consideradas como servidores públicos para efecto de esta Ley y, en consecuencia, son susceptibles de incurrir en la actuación bajo conflicto de interés, ya que por regla general, las actividades que desempeñan tienen como naturaleza el «servicio a la sociedad» (Tesis Aislada 2ª. XCIII/2006, N. ${ }^{\circ}$ 173672).

\section{b) Presencia de un interés}

Al hablar de la presencia de un interés, nos referimos a aquella situación o situaciones en que el servidor público tiene inclinación del ánimo hacia una persona - física o moral-, objeto o circunstancia que puede tener como consecuencia que su discernimiento sea menos confiable de lo que sería habitualmente.

Estos intereses son innumerables y de gran subjetividad, ya que varían de persona a persona, pudiendo consistir en cualquier influencia que «tienda a hacer menos confiable el juicio profesional del que sería habitualmente, porque podría conllevar algún tipo de beneficio o ventaja personal o profesional, directa o indirecta, presente o futura, pecuniaria o de otra naturaleza» (Parlamento de Cataluña, 2016, p. 30).

Remitiéndonos a la definición de conflictos de intereses que aporta la Ley General de Responsabilidades Administrativas, los intereses que afectan el desempeño de las funciones de los servidores públicos, pueden ser: personales, familiares o de negocios. En este punto particular, la complejidad a que nos enfrentamos es que la disposición en estudio no establece lo que debe entenderse por cada uno de estos tipos de intereses. 
De estos tres tipos, el que menor complejidad representa es el interés familiar, ya que para comprenderlo basta con determinar lo que es la familia como institución y quiénes la conforman. La Real Academia Española (s.f.) define a la familia como el «conjunto de ascendientes, descendientes, colaterales y afines de linaje», en el mismo sentido, la jurisprudencia reconoce que el derecho de la familia está dirigido a delimitar las relaciones conyugales, de concubinato y parentesco, conformadas por un sistema especial de deberes y facultades entre padres e hijos, consortes y parientes (Jurisprudencia I.5‥C.J/11, N. ${ }^{\circ} 162604$ ).

Por lo tanto, se considera que existe un interés familiar cuando la atención, tramitación o resolución de un asunto competencia del servidor público está relacionada directamente con una persona con quien tiene un vínculo familiar (cónyuge, concubino o concubinaria, parientes consanguíneos, por afinidad o civiles). Ahora bien, por lo que se refiere a la limitante de grado, actualmente no hay una disposición vigente que la establezca; por lo que, aplicando la máxima «Ubi lex non distinguit, nec nos distinguere debemus», es posible considerar que, incluso del parentesco en línea transversal, en décimo grado, deriva un interés familiar ${ }^{[3]}$.

\section{ii) El interés de negocios}

En lo que respecta al interés de negocios, lo entendemos de manera habitual como aquellos intereses que son objeto o materia de ocupación lucrativa para el servidor público; se refiere particularmente a los asuntos de las personas servidoras públicas o su círculo de intereses, que se relacionen con actividades profesionales, laborales o comerciales, incluyendo socios o sociedades de las que el servidor público o las personas de su círculo formen parte.

\section{iii) El interés personal}

La noción de interés personal tampoco es fácil de delimitar. Ciertamente, los intereses familiares o de negocios no nos plantean ninguna duda, pero también constituyen intereses particulares el amor, la amistad o la gratitud; por eso está bastante aceptado que las conexiones familiares son una de las fuentes más comunes de conflictos de interés. En definitiva, podríamos entender que un interés personal es cualquier influencia, lealtad, emoción u otra característica de una situación que tienda a hacer menos confiable el juicio profesional del que sería habitualmente, porque podría conllevar algún tipo de beneficio o ventaja personal o profesional, directa o indirecta, presente o futura, pecuniaria o de otra naturaleza.

En este supuesto, los intereses personales que afectan el ejercicio de la actividad del servidor público resultan ser un aspecto completamente casuístico que deberá ser valorado por el propio servidor público para abstenerse de intervenir, y en caso de ser denunciado, se tendrá que argumentar y demostrar que un interés de este tipo afectó la objetividad e imparcialidad en la decisión adoptada por el servidor público.

\section{c) Actuación en representación}

Para comprender este punto particular, resulta necesario hacer un brevísimo repaso a la denominada «Teoría del órgano». Esta teoría, a través de la equiparación del Estado

[3] El artículo 8, fracción XI, de la Ley Federal de Responsabilidades Administrativas de los Servidores Públicos, vigente hasta el 18 de julio de 2017, establecía como limitante de parentesco el cuarto grado en línea recta. 
a un órgano, de creación jurídica abstracta, busca explicar la relación existente entre el Estado y sus entes auxiliares jurídicamente personificados, que son entes -también abstractos-que actúan por medio de persona físicas -servidores públicos-.

El principal exponente mexicano en dicho tema es Andrés Serra Rojas (1964), quien señala que el órgano se integra por dos elementos: los servidores públicos que tienen a su cargo cumplir con los mandatos de la ley en el ámbito de su competencia y el ente formado con la competencia, los derechos, deberes, obligaciones, posibilidades y limitaciones vinculadas con una finalidad específica; en este sentido, cuando las personas físicas adoptan el carácter de servidores públicos, realizan actos por cuenta y nombre de la administración pública, investidos de atributos especiales que les confiere el derecho público.

Así las cosas, cuando los servidores públicos desempeñan sus funciones, no actúan en calidad de personas privadas, sino en representación del Estado y, como consecuencia, de los ciudadanos que legítimamente confían en su buen juicio, convirtiéndose entonces en depositarios de la confianza del Estado y de la ciudadanía (OCDE, 2005, p. 15).

Es por este motivo que los conflictos de intereses son un problema real, porque los servidores públicos emiten un juicio profesional en nombre del Estado y la ciudadanía.

\section{d) Discernimiento}

Conforme al principio de legalidad, que coloquialmente se enuncia como «los particulares pueden hacer todo aquello que no está prohibido, las autoridades sólo pueden hacer lo que la ley les permite (Acción de Inconstitucionalidad 26/2006, promovida por los Senadores integrantes de la quincuagésima novena legislatura del Congreso de la Unión.) las autoridades del Estado sólo pueden actuar en la forma y en los términos que establece la normativa; por lo tanto, únicamente pueden ejercer las fa- cultades y atribuciones previstas expresamente en la en la ley que regula sus actos.

En tal sentido, el elemento del discernimiento es una facultad que deriva directamente de las funciones y atribuciones otorgadas al servidor público en virtud de su empleo, cargo o comisión. Se refiere al ejercicio que realiza el servidor público en el desempeño de sus funciones, es decir, el juicio analítico que se exige al servidor público para tomar una decisión correcta a partir de un sentido de conocimiento, destreza o perspicacia (Parlamento de Cataluña, 2016, p. 30).

Así las cosas, se trata de un elemento determinante en la actuación bajo conflicto de interés, puesto que, podremos hablar de la configuración de dicha falta administrativa únicamente en aquellos casos en que el servidor público que manifiesta el interés personal, familiar o de negocios, esté dotado de la facultad de decidir sobre la atención, tramitación o resolución del asunto en cuestión, pues de ello dependerá la generación de beneficios a él mismo o a terceros.

Ahora bien, en el supuesto de que el servidor público involucrado tenga un vínculo del que derive un interés personal, familiar o de negocios y, sin embargo, no cuente con la atribución de ejercer su juicio en el asunto concreto, entonces no se configuraría la actuación bajo conflicto de interés, sino otras faltas administrativas graves asociadas, como lo son el tráfico de influencias, uso de indebido de información, entre otras.

\section{e) Afectación del ejercicio de funciones}

El artículo 109 Constitucional, fracción III (1917) y el primer párrafo del artículo 7, de la Ley General de Responsabilidades Administrativas, establecen que el actuar de los servidores públicos debe estar orientado por los principios de legalidad, honradez, lealtad, imparcialidad y eficiencia y, la inobservancia de ellos puede derivar en la comisión de una falta administrativa. 
En lo que se refiere a «afectación», la Real Academia Española (s.f.) lo define con los adjetivos menoscabar, perjudicar e influir desfavorablemente; en este sentido, la afectación del ejercicio de funciones del servidor público se encuentra directamente relacionada con el elemento del discernimiento y la presencia de un interés, ya que se produce en aquellos casos en que existen factores externos que nublan el juicio de que deberá hacer uso. Vulnerando, especialmente, el principio de imparcialidad.

\section{f) Beneficio o ventaja en virtud del interés involucrado}

Por lo que respecta al beneficio o ventaja, se trata de una consecuencia de la actuación bajo Conflicto de Interés, más que de un elemento en sí mismo; en el entendido de que el conflicto de interés es una «posible afectación del desempeño imparcial y objetivo de las funciones de los Servidores Públicos» (Ley General de Responsabilidades Administrativas, Artículo 3, fracción VI), es meramente una situación de riesgo hasta en tanto no se configure la falta administrativa.

Una vez que el servidor público se halla ante la presencia de un conflicto de intereses (situación de riesgo), y a pesar de ello decide actuar (actuación bajo conflicto de interés), es cuando se actualiza el supuesto y surge el beneficio o ventaja, que puede ser «para sí mismo, su cónyuge, parientes consanguíneos, parientes civiles o para terceros con los que tiene relaciones profesionales, laborales o de negocios, o socios o sociedades de las que el servidor público o las personas referidas formen parte» (Ley General de Responsabilidades Administrativas, Artículo 52).
De lo anterior se observa que el beneficio o ventaja resultado de la actuación bajo conflicto de interés puede consistir en cualquier mejora, privilegio, favor, servicio, ganancia, descuento, concesión, prerrogativa, etc., y no únicamente para el servidor público, sino también para aquellas personas con quienes tiene algún vínculo que da origen a la situación de riesgo.

No obstante, la vigente Ley General de Responsabilidades Administrativas es omisa en cuanto a establecer el «beneficio» dentro del tipo administrativo, abriendo la posibilidad a que, incluso en aquellos casos en que el servidor público intervenga en un asunto de su interés sin obtener beneficios, se configure la falta administrativa. Lo que sería congruente con el espíritu de la ley.

\section{EL MARCO JURÍDICO MEXICANO}

En lo que corresponde a la regulación, se presentan las leyes que contienen obligaciones para los servidores públicos de la Administración Pública Federal, respecto a conflictos de intereses; si bien es cierto, en la mayoría de las disposiciones no se emplea expresamente el término, también lo es que las conductas a que se hace referencia coinciden con las características de dicho concepto.

Cabe resaltar que, a excepción de la Ley General de Responsabilidades Administrativas, que es la legislación encargada de determinar las faltas administrativas en que pueden incurrir los servidores públicos, y la Ley Federal de Austeridad Republicana, las leyes a que se hará referencia corresponden a lo que se denomina como «zonas de riesgo» ${ }^{[4]}$, pues atañen a procedimientos en los que se tiene manejo de recursos públicos y mayor contacto con particulares.

[4] La Secretaría de la Función Pública define a las zonas de riesgo como «las zonas más propensas a riesgos contra la integridad» y refiere que la identificación de estas áreas permite llevar a cabo un primer nivel de control para disipar las dudas o confirmar la existencia de conflictos de intereses. Se consideran zonas de riesgo las siguientes: Adquisiciones, Obra Pública, Recursos Financieros, Recursos Humanos, Recursos Materiales, Tecnologías de la Información, Transparencia, Auditoría y Control Interno. 


\subsection{La Ley General de Responsabilidades Ad- ministrativas}

Derivado de la reforma constitucional en materia de combate a la corrupción, publicada en el Diario Oficial de la Federación el 23 de mayo de 2015, se adicionó la facultad del Congreso de la Unión para expedir la ley general en materia de responsabilidades administrativas de los servidores públicos y las que correspondan a los particulares vinculados. Bajo este mandato constitucional, el 18 de julio de 2016 se publicó la Ley General de Responsabilidades Administrativas, vigente a partir del 19 de julio de 2017.

Esta Ley es de orden público y tiene por objeto distribuir competencias entre los tres órdenes de gobierno, a efecto de establecer las responsabilidades administrativas de los servidores públicos, sus obligaciones, las sanciones aplicables por los actos $u$ omisiones en que éstos incurran; destacando como novedad que, también establece sanciones para los particulares vinculados con faltas administrativas graves (Artículo 1).

Atendiendo a dicho fin, establece que el Conflicto de Interés debe entenderse como «la posible afectación del desempeño imparcial y objetivo de las funciones de los Servidores Públicos en razón de intereses personales, familiares o de negocios» (Artículo3, fracción VI).

De ahí que, el elemento característico de los conflictos de intereses se vincule con la confrontación entre los intereses privados del servidor público y los correspondientes a las funciones de su empleo, cargo o comisión, teniendo como consecuencia la afectación de su desempeño imparcial.

En tal sentido, esta Ley establece la falta administrativa grave «actuación bajo conflicto de interés", señalando que incurre en este supuesto «el servidor público que intervenga por motivo de su empleo, cargo o comisión en cualquier forma, en la atención, tramitación o resolución de asuntos en los que tenga Conflicto de Interés o impedimento legal» (Artículo 58).
Desde esta perspectiva, debe advertirse que el conflicto de interés por sí mismo no constituye una falta administrativa, ya que la conducta que resulta sancionable es la actuación bajo conflicto de interés; es decir, que el servidor público, por motivo de su empleo, cargo o comisión, decida intervenir de cualquier forma, en algún asunto en el que tenga un conflicto de interés o impedimento legal.

Asimismo, se determinan las medidas que deben ser adoptadas en el supuesto de encontrarse ante un conflicto de interés (Artículo 58):

\section{[...]}

Al tener conocimiento de los asuntos mencionados en el párrafo anterior, el servidor público informará tal situación al jefe inmediato o al órgano que determine las disposiciones aplicables de los entes públicos, solicitando sea excusado de participar en cualquier forma en la atención, tramitación o resolución de los mismos.

Será obligación del jefe inmediato determinar y comunicarle al servidor público, a más tardar 48 horas antes del plazo establecido para atender el asunto en cuestión, los casos en que no sea posible abstenerse de intervenir en los asuntos, así como establecer instrucciones por escrito para la atención, tramitación o resolución imparcial y objetiva de dichos asuntos.

Ahora bien, a partir de la publicación el 19 de noviembre de 2019, del Decreto por el que se expide la Ley Federal de Austeridad Republicana; y se reforman y adicionan diversas disposiciones de la Ley General de Responsabilidades Administrativas y de la Ley Federal de Presupuesto y Responsabilidad Hacendaria, entre otras modificaciones, se amplió la falta grave "contratación indebida» y se incluyó el tipo administrativo «nepotismo».

Bajo este contexto, dentro de la falta administrativa "contratación indebida», se adicionó el párrafo segundo (Artículo 59), para disponer: 
Incurrirá en la responsabilidad dispuesta [...], el servidor público que intervenga o promueva, por sí o por interpósita persona, en la selección, nombramiento o designación de personas para el servicio público en función de intereses de negocios.

Del párrafo incorporado, se observa la inclusión de los intereses de negocios como elemento determinante en la selección, nombramiento o designación del servidor público; vínculo que, sumado a la acción de intervenir o promover, da lugar a la configuración de la falta administrativa grave «contratación indebida».

De acuerdo con la conducta adicionada al tipo administrativo contratación indebida, se observa que la misma se encuentra igualmente tipificada con la actuación bajo conflicto de interés en razón de intereses de negocios, al momento en el que el servidor público responsable de la contratación interviene, de cualquier forma, en la atención, tramitación o resolución de algún asunto en el que tiene un conflicto de interés en razón de intereses de negocios.

Por otro lado, se agregó el artículo 63 Bis, en el cual se contempla la falta administrativa grave «nepotismo», que dispone:

Cometerá nepotismo el servidor público que, valiéndose de las atribuciones o facultades de su empleo, cargo o comisión, directa o indirectamente, designe, nombre o intervenga para que se contrate como personal de confianza, de estructura, de base o por honorarios en el ente público en que ejerza sus funciones, a personas con las que tenga lazos de parentesco por consanguinidad hasta el cuarto grado, de afinidad hasta el segundo grado, o vínculo de matrimonio o concubinato.

Este artículo en particular se refiere específicamente a aquellos supuestos de contratación de personal en el que un servidor público interviene, existiendo una relación de parentesco por consanguinidad o afinidad, independientemente del tipo de contratación de que se trate.

\subsection{Ley Federal de Austeridad Republicana}

La Ley Federal de Austeridad Republicana, publicada en el Diario Oficial de la Federación el 19 de noviembre de 2019, tiene como objeto regular y normar las medidas de austeridad que deben observarse en el ejercicio del gasto público federal y coadyuvar a que los recursos económicos se administren con eficacia, eficiencia, economía, transparencia y honradez (Artículo 1); además, es importante destacar que sus disposiciones son aplicables a todas las dependencias, entidades, organismos y demás entes que integran la Administración Pública Federal.

Entre las conductas reguladas en esta ley, se destacan obligaciones para los servidores públicos relevantes para la regulación de los conflictos de intereses, entre ellas, la prohibición del nepotismo, la obligación de separarse de los intereses legales y la prohibición para ocupar puestos en empresas en un plazo menor a diez años.

Su artículo 24 dispone obligaciones de las personas que pretendan desempeñar un empleo, cargo o comisión respecto a los conflictos de intereses, en el sentido siguiente:

Para ocupar cualquier empleo, cargo o comisión en algún ente público, las personas interesadas se verán obligadas a separarse legalmente de los activos e intereses económicos particulares que estén relacionados con la materia o afecten de manera directa el ejercicio de sus responsabilidades públicas, y que signifiquen un conflicto de interés conforme a lo establecido en la Ley General de Responsabilidades Administrativas.

Los servidores públicos comprendidos en los grupos jerárquicos de mando superior a que se refiere el manual de percepciones previsto en la Ley Federal de Presupuesto y Responsabilidad Hacendaria del Gobierno Federal, que por cualquier motivo se separen de su cargo, no podrán ocupar puestos en empresas que hayan supervisado, regulado o respecto de las cuales hayan tenido 
información privilegiada en el ejercicio de su cargo público, salvo que hubiesen transcurrido al menos diez años.

En este punto, destaca que la obligación de las personas que pretenda ocupar un empleo, cargo o comisión a separarse de los activos e intereses económicos particulares que puedan afectar el desempeño de sus funciones; así como el nepotismo, fueron adicionados de manera particular en los artículos 7 y 63 de la LGRA; sin embargo, en lo que respecta a las obligaciones post-empleo de los servidores públicos de mando superior, la reforma a la Ley General de Responsabilidades Administrativas no regula tal conducta, por lo que existe un vacío jurídico que impide su sanción.

\subsection{La Ley Federal de Procedimientos Admi- nistrativos}

Publicada en el Diario Oficial de la Federación el 4 de agosto de 1994 y reformada por última vez el 18 de mayo de 2018, tiene por objeto regular los actos, procedimientos y resoluciones de la Administración Pública Federal centralizada, sin que resulte aplicable a las materias de carácter fiscal, responsabilidades de los servidores públicos, justicia agraria y laboral, ni al ministerio público en ejercicio de sus funciones constitucionales (Artículo 1).

Si bien, esta Ley no es aplicable a la materia de responsabilidades de los servidores públicos, resulta relevante para el objeto de estudio de la materia que nos ocupa, toda vez que establece limitantes a los servidores públicos para conocer de procedimientos cuando tengan un interés, y la obligación de excusarse cuando se ubiquen en dicho supuesto.

En tal sentido, se considera que existe un impedimento cuando el servidor público tiene interés directo o indirecto en el asunto de que se trate o en otro semejante; tiene interés su cónyuge o sus parientes, ya sean consanguíneos o colaterales; hubiere parentesco de consanguinidad o de afinidad, con cualquiera de los interesados, con los administradores de entidades o sociedades interesadas o con los mandatarios que intervengan en el procedimiento; exista amistad o enemistad manifiesta que se hagan patentes mediante hechos o actitudes evidentes; intervenga como perito o como testigo en el asunto de que se trata o; tenga relación de servicio con las personas interesadas directamente en el asunto (Artículo 21).

Al encontrarse en alguna de dichas circunstancias, el servidor público debe excusarse tan pronto tenga conocimiento y lo comunicará a su superior inmediato, quien resolverá lo conducente; cuando hubiere otro servidor público con competencia le será turnado, en caso contrario, quien se hubiere excusado deberá atenderlo bajo la supervisión de su superior jerárquico (Artículo 22).

\subsection{La Ley de Adquisiciones, Arrendamientos y Servicios del Sector Público}

Esta Ley, publicada en el Diario Oficial de la Federación el 4 de enero de 2000 y reformada por última vez el 10 de noviembre de 2014, tiene como objeto reglamentar la aplicación del artículo 134 de la Constitución Política de los Estados Unidos Mexicanos (1917), en materia de adquisiciones, arrendamientos de bienes muebles y prestación de servicios de cualquier naturaleza.

Lo anterior, respecto de las unidades administrativas de la Presidencia de la República; las Secretarías de Estado y la Consejería Jurídica del Ejecutivo Federal; la Fiscalía General de la República; los organismos descentralizados; las empresas de participación estatal mayoritaria y los fideicomisos en los que el fideicomitente sea el gobierno federal o una entidad paraestatal; las entidades federativas; los municipios y los entes públicos, con cargo total o parcial a recursos federales, conforme a los convenios que celebren con el Ejecutivo Federal (Artículo 1).

La razón de que se incluya como parte del marco jurídico que regula los conflictos de intereses, es que establece limitantes a las dependencias y entidades para recibir o adjudicar 
contratos, con personas respecto de quienes se derive la existencia de un interés. Particularmente, dispone en su Artículo 50 lo siguiente:

Las dependencias y entidades se abstendrán de recibir proposiciones o adjudicar contrato alguno en las materias a que se refiere esta Ley, con las personas siguientes:

I. Aquéllas en que el servidor público que intervenga en cualquier etapa del procedimiento de contratación tenga interés personal, familiar o de negocios, incluyendo aquéllas de las que pueda resultar algún beneficio para él, su cónyuge o sus parientes consanguíneos hasta el cuarto grado, por afinidad o civiles, o para terceros con los que tenga relaciones profesionales, laborales o de negocios, o para socios o sociedades de las que el servidor público o las personas antes referidas formen o hayan formado parte durante los dos años previos a la fecha de celebración del procedimiento de contratación de que se trate;

II. Las que desempeñen un empleo, cargo o comisión en el servicio público, o bien, las sociedades de las que dichas personas formen parte, sin la autorización previa y específica de la Secretaría de la Función Pública;

[...]

De conformidad con esta disposición normativa, existe una prohibición directa a las dependencias y entidades a no recibir proposiciones o adjudicar contratos a servidores públicos que intervengan en los procedimientos de contrataciones; así como también, a empresas en las que formen parte servidores públicos y no cuenten con la autorización específica ante el titular del órgano interno de control en la dependencia o entidad respectiva.

Es importante destacar que lo previsto en el artículo 50, fracción I, de la LAASSP, desarrolla los conflictos de intereses respecto a las personas que puede ser beneficiadas; situación que no se realiza de manera específica en la LGRA.

\subsection{La Ley de Obras Públicas y Servicios Rela- cionados con las Mismas}

La Ley de Obras Públicas y Servicios Relacionados con las Mismas, se publicó el 4 de enero de 2000, en la misma fecha que la LAASSP y, el 13 de enero de 2016 fue reformada por última vez.

Tiene como objeto reglamentar la aplicación del artículo 134 de la Constitución Política de los Estados Unidos Mexicanos, en materia de contrataciones de obras públicas, así como de los servicios relacionados con las mismas, que realicen las unidades administrativas de la Presidencia de la República; las Secretarías de Estado y la Consejería Jurídica del Ejecutivo Federal; la Fiscalía General de la República; los organismos descentralizados; las empresas de participación estatal mayoritaria y los fideicomisos en los que el fideicomitente sea el Gobierno Federal o una entidad paraestatal, y las entidades federativas, los municipios y los entes públicos, con cargo total o parcial a recursos federales, conforme a los convenios que celebren con el Ejecutivo Federal (Artículo 1).

De este instrumento normativo, el artículo que resulta relevante para la materia en estudio es el 51, fracciones I y II, en virtud de que establece a las dependencias y entidades, la prohibición para recibir propuestas o adjudicar contratos, con personas respecto de las que se derive la existencia de un conflicto de interés, como a continuación se cita:

Artículo 51. Las dependencias y entidades se abstendrán de recibir proposiciones $o$ adjudicar contrato alguno en las materias a que se refiere esta Ley, con las personas siguientes:

I. Aquéllas en que el servidor público que intervenga en cualquier etapa del procedimiento de contratación tenga interés personal, familiar o de negocios, incluyendo aquellas de las que pueda resultar algún beneficio para él, su cónyuge o sus parientes consanguíneos hasta el cuarto grado, por afinidad o civiles, o 
para terceros con los que tenga relaciones profesionales, laborales o de negocios, o para socios o sociedades de las que el servidor público o las personas antes referidas formen o hayan formado parte durante los dos años previos a la fecha de celebración del procedimiento de contratación de que se trate;

II. Las que desempeñen un empleo, cargo o comisión en el servicio público, o bien, las sociedades de las que dichas personas formen parte, sin la autorización previa y específica de la Secretaría de la Función Pública;

\section{$[\ldots]$}

Desde esta perspectiva, al igual que la LAASSP, el artículo 51 de la LOPSRM, dispone normas de gran relevancia para los conflictos de intereses, y desarrolla esta conducta respecto a las personas que puede ser beneficiadas; situación que no se realiza de manera específica en la LGRA.

\subsection{Ley de Asociaciones Público Privadas}

La Ley de Asociaciones Público Privadas, publicada en el Diario Oficial de la Federación el 16 de enero de 2012, fue reformada por última ocasión el 15 de junio de 2018; esta norma tiene por objeto regular los esquemas para el desarrollo de proyectos de asociaciones público-privadas, bajo los principios de los artículos 25 y 134 Constitucionales (Artículo 1).

En el mismo sentido que la LAASSP y la LOPSRM, establece limitantes para la participación de personas con quienes se presume la existencia de un conflicto de interés en los concursos y adjudicaciones para desarrollar proyectos, como a continuación se señala:

Artículo 42. No podrán participar en los concursos, ni recibir adjudicación para desarrollar un proyecto de asociación públicoprivada, las personas siguientes:

I. Aquellas en las que algún servidor público que intervenga en cualquier etapa del procedimiento de contratación tenga interés personal, familiar o de negocios, o bien de las que pueda resultar algún beneficio para él, su cónyuge o sus parientes consanguíneos o por afinidad hasta el cuarto grado, o civil, o para terceros con los que tenga relaciones profesionales, laborales o de negocios, o para socios o sociedades de las que el servidor público o las personas antes referidas formen o hayan formado parte durante los dos años previos a la fecha de celebración del procedimiento de contratación de que se trate; [...]

\subsection{Protocolo de Actuación en materia de Contrataciones Públicas, otorgamiento y prorroga de Licencias, Permisos, Autori- zaciones y Concesiones}

El 20 de agosto de 2015, se publicó en el Diario Oficial de la Federación el Protocolo de actuación en materia de Contrataciones Públicas, Otorgamiento y Prórroga de Licencias, Permisos, Autorizaciones y Concesiones, y fue reformado mediante las publicaciones de 19 de febrero de 2016 y 28 de febrero de 2017.

Este documento busca promover las mejores prácticas en materia de combate a la corrupción y prevención de conflictos de intereses, y fomenta la corresponsabilidad entre ciudadanía y gobierno para el fortalecimiento de la ética y la integridad.

En consecuencia, tiene por objeto expedir las reglas que deben observar, en su contacto con particulares, los servidores públicos inscritos en el «Registro de Servidores públicos de la Administración Pública Federal que intervienen en procedimientos de contrataciones públicas, el otorgamiento de licencias, permisos, concesiones y autorizaciones, así como en la enajenación de bienes muebles de la administración pública federal y en la asignación y emisión de dictámenes en materia de avalúos y justipreciación de rentas»; así como los servidores públicos que fungen como residentes de obra, en su trato con los superintendentes de construcción (Artículo Primero). 
Por otro lado, establece mecanismos para que los particulares puedan formular un manifiesto de vínculos o relaciones, que tengan con servidores públicos de alto nivel.

\section{DEBILIDADES EN LA REGULACIÓN DE LOS CONFLICTOS DE INTERESES}

De la revisión realizada en el apartado anterior, se desprende que las principales dificultades a que nos enfrentamos, tratándose de los conflictos de intereses, son la indeterminación jurídica y la normalización de los intereses personales.

\subsection{La indeterminación jurídica}

Como bien lo señaló Góngora Pimentel (2008), el derecho administrativo sancionador estudia lo relativo a la potestad sancionadora de la administración, que implica la acción punitiva del Estado y que, si bien difiere de la derivada el derecho penal, ello no excluye la adopción de diversos principios propios de esta última disciplina. Bajo esta consideración, es posible precisar que los principios de legalidad, tipicidad, presunción de inocencia, non bis in ídem, debido proceso, entre otros, también resultan aplicables al derecho administrativo sancionador, aunque no de forma limitativa.

En este sentido, es posible referirnos a la Ley General de Responsabilidades Administrativas, como una norma de derecho administrativo sancionador ya que, dentro del procedimiento convergen como características principales (Tesis Aislada 1‥ XXXV/2017):

1. Presupone la existencia de un tipo administrativo que conlleva al surgimiento de una infracción, la responsabilidad administrativa.

2. Se sigue en forma de juicio, en el que se determina si la conducta del servidor público contraviene aquellas prohibiciones a las cuales se sujeta el ejercicio de su función.

3. Tiene por finalidad procurar la correcta actuación de los servidores públicos, sancionar a los infractores y, en su caso, lograr la restitución de aquellos bienes jurídicos que fueron afectados con su irregular actuación.

Las anteriores consideraciones resultan de especial atención en virtud de que, al hablar de derecho administrativo sancionador se abre la obligatoriedad de las autoridades a respetar los principios jurídicos aplicables a esa materia.

En consecuencia, atendiendo a lo señalado en la jurisprudencia «Tipicidad. El principio relativo, normalmente referido a la materia penal, es aplicable a las infracciones y sanciones administrativas», en la interpretación constitucional de los principios del derecho administrativo sancionador debe acudirse al principio de tipicidad, de modo tal que si cierta disposición administrativa establece una sanción por alguna infracción, la conducta realizada por el afectado debe encuadrar exactamente en la hipótesis normativa previamente establecida, sin que sea lícito ampliar ésta por analogía o por mayoría de razón (Jurisprudencia 100/2006).

Entonces, para que la conducta del servidor público sea encuadrada en el tipo administrativo «actuación bajo conflicto de interés», es necesario que se reúna cada una de las características establecidas en el silogismo normativo, a saber:

I. El involucrado es un servidor público.

II. Interviene por motivo de su empleo, cargo o comisión, de cualquier forma, en la atención, tramitación o resolución de asuntos.

III. Tiene conflicto de interés o impedimento legal.

Hasta este punto parece sencillo, sin embargo, ya que nos remitimos a la definición de conflicto de interés, tenemos que éste puede ser causado por la existencia de un interés personal, familiar o de negocios, conceptos que se encuentran jurídicamente indeterminados, podemos hacer una labor de interpretación 
sistemática para comprender lo referente al interés familiar y de negocios; sin embargo, el interés personal seguirá en duda: ¿Cuándo hablamos de un interés personal?

\subsection{La normalización de los intereses per- sonales}

En la práctica cotidiana del servicio público, el interés que con mayor frecuencia afecta la toma de decisiones de los funcionarios, es el interés personal, por dos razones principales:

1) Se trata de una práctica que históricamente ha viciado el ejercicio de la función pública, beneficiando a los círculos cercanos de quienes ejercen el poder.

2) A partir de la práctica reiterada, se volvió una conducta social y moralmente aceptada, tanto por los servidores públicos mismos como por la ciudadanía.

Ello tiene como consecuencia que, ante esta falta administrativa las actitudes de los sujetos involucrados y perjudicados sean muy diversas, pudiendo ir desde la justificación de dichas conductas hasta su aceptación, simplemente porque «así funciona».

En el primer apartado, en la búsqueda de determinar esta situación coyuntural, se propuso como concepto del interés personal: cualquier influencia, lealtad, emoción u otra característica de una situación que tienda a hacer menos confiable el juicio profesional del que sería habitualmente, porque podría conllevar algún tipo de beneficio o ventaja personal o profesional, directa o indirecta, presente o futura, pecuniaria o de otra naturaleza.

Sin embargo, se trata de un problema de amplio alcance y, en cierto modo inevitable, el gran reto en esta materia es que comúnmente se sostiene que no tiene sentido preocuparse por su dimensión ética, pero esta suele ser una actitud relativista, desmentida, evidentemente, por los hechos.

\section{CONSIDERACIONES FINALES}

El primer paso para la erradicación de la actuación bajo conflicto de interés, por intereses personales de los servidores públicos, es reformar la Ley General de Responsabilidades Administrativas, precisando el concepto de «conflictos de intereses» e incluyendo las definiciones de «intereses personales», «intereses familiares» e «intereses de negocios»; de tal manera que el tipo administrativo permita identificar claramente, las conductas que encuadran en dicha hipótesis normativa.

Una vez determinados los conceptos jurídicos anteriores, será más asequible la adecuación de las conductas a la falta administrativa «actuación bajo conflicto de interés» $y$, en consecuencia, su sanción.

No obstante, en la práctica se constata que la existencia de conflictos de intereses en la toma de decisiones de los servidores públicos son conductas reiteradas en el paso del tiempo, que se han adoptado como normales en la Administración Pública, percibiéndose las medidas de prevención como meras obligaciones que hay que cumplir, en lugar de como instrumentos para asegurar la objetividad e imparcialidad de los servidores públicos.

Por este motivo, adicionalmente a la determinación jurídica de los conceptos indeterminados, la prevención y resolución de los conflictos de interés forma parte de una responsabilidad educativa mayor, por parte de las instituciones encargadas de atender cuestiones éticas en la Administración Pública.

El objetivo no es únicamente mantener a los servidores públicos lo suficientemente informados para protegerlos de posibles conflictos de intereses, también es necesario adoptar una cultura de integridad y comprender que las regulaciones éticas y mecanismos de control son parte sustancial del aparato gubernamental para mantener la confianza pública, para proteger la toma de decisiones y mejorar el funcionamiento del sistema democrático. 


\section{REFERENCIAS}

Arellano Gault, David. ¿Podemos reducir la corrupción en México? Límites y posibilidades de los instrumentos a nuestro alcance, Centro de Investigación y Docencia Económicas, Segunda Edición, México, 2018.

Bautista, Oscar Diego. Cien años de corrupción en México a partir de la era postrevolucionaria 1917-2017, Colección de Cuadernos para prevenir y evitar la corrupción, Poder Legislativo del Estado de México, Tomo 2, México, 2017.

Brioschi, Carlo Alberto. Breve historia de la corrupción, de la antigüedad a nuestros días, Taurus, España, 2019.

Dussauge Laguna, Mauricio I. «Combate a la Corrupción y rendición de cuentas: avances, limitaciones, pendientes y retrocesos», Los Grandes problemas de México, Vol. XIII Políticas Públicas, COLMEX, México, 2010, págs. 207-252.

Fernández Ruiz, Jorge. Derecho Administrativo y Administración Pública, Séptima edición, México: Porrúa, 2016.

Góngora Pimentel, Genaro David. El reconocimiento del Derecho Administrativo Sancionador en la Jurisprudencia Constitucional Mexicana, México: Biblioteca Jurídica Virtual del Instituto de Investigaciones Jurídicas de la UNAM, p. 255-274, 2008.

Holmes, Leslie. ¿Qué es la corrupción?, Grano de Sal, México, 2019.

Krauze, Enrique. Por una democracia sin adjetivos, Debate, México, 2016.

OCDE. Managing Conflict of Interest in the Public Sector a Toolkit, Francia, 2005. https:// read.oecd-ilibrary.org/governance/managing-conflict-of-interest-in-the-public-sector_9789264018242-en\#page9

OCDE. Recomendación del Consejo de la OCDE sobre Integridad Pública, 2017. http://www. oecd.org/gov/integridad/recomendacionintegridad-publica/
Parlamento de Cataluña, La gestión de los conflictos de interés en el sector público de Cataluña, Barcelona, 2016. https://www.parlament.cat/document/cataleg/182556.pdf

Rocha Díaz, Salvador. «Las reformas constitucionales iniciadas por el Licenciado Miguel de la Madrid Hurtado», México: Biblioteca Jurídica Virtual del Instituto de Investigaciones Jurídicas de la UNAM, 1986. https://revistas-colaboracion.juridicas.unam.mx/index.php/rev-facultad-derecho-mx/article/view/27619/24948

Rodríguez-Arana, Jaime. La dimensión ética de la función pública, Instituto Nacional de Administración Pública, Madrid, 2013.

Rose-Ackerman, Susan. La corrupción y los gobierno. Causas, consecuencias y reforma. Siglo XXI de España Editores, España, 2000.

Secretaría de la Función Pública, Conceptos Fundamentales sobre Conflicto de Intereses, Secretaría de la Función Pública, México, 2018.

Secretaría de la Función Pública, Guía para prevenir la actuación bajo Conflicto de Intereses, 2nda edición, Secretaría de la Función Pública, México 2017. https://www.gob.mx/ cms/uploads/attachment/file/241091/Guia Conflictos_de_Interes_LGRA_v2.pdf

Secretaría de la Función Pública, Diagnóstico sobre Procedimientos Iniciados para Investigar y Sancionar Conflictos de Intereses. Abril 2002-Julio 2017, Secretaría de la Función Pública, México, 2018. https://www. gob.mx/sfp/documentos/diagnostico-sobreprocedimientos-iniciados-para-investigar$y$-sancionar-conflictos-de-intereses-abril2002-julio-2017

Serra Rojas, Andrés. Teoría General del Estado, Porrúa, México, 1964.

Serrano Sánchez, Jesús Antonio. Ética en el margen de las políticas públicas, Comisión de Vigilancia de la Auditoría Superior de la Federación, México, 2018.

Suprema Corte de Justicia de la Nación, Pleno, Jurisprudencia 100/2006, Registro 174326, 
tipicidad. El principio relativo, normalmente referido a la materia penal, es aplicable a las infracciones y sanciones administrativas, Semanario Judicial de la Federación y su Gaceta, Tomo XXIV.

Suprema Corte de Justicia de la Nación, Primera Sala, Tesis Aislada 1. XXXV/2017, Registro 2013954, derecho administrativo sancionador. Concepto de sanción que da lugar a su aplicación, Gaceta del Semanario Judicial de la Federación, Libro 40, Tomo I.

Suprema Corte de Justicia de la Nación, Segunda Sala, Tesis Aislada XCIII/2006, N. ${ }^{\circ} 173672$, servidores públicos. el artículo 108, primer párrafo, de la Constitución Política de los Estados Unidos mexicanos, no es limitativo sino enunciativo, Semanario Judicial de la Federación y su Gaceta, Tomo XXIV.

Tribunales Colegiados de Circuito, Quinto Tribunal Colegiado en Materia Civil del Primer Circuito, Jurisprudencia I.5o.C.J/11, N. ${ }^{\circ}$ 162604, derecho de familia. Su concepto, Semanario Judicial de la Federación y su Gaceta, Tomo XXXIII.

Zaid, Gabriel. «La propiedad privada de las funciones públicas», Vuelta, N. ${ }^{\circ} 120$, México, 1986, pp. 25-32.

Zaid, Gabriel. El poder corrompe, Debate, México, 2019.

Zaldívar, Oscar. «Los Conflictos de Intereses», Moneda, N. ${ }^{\circ}$ 146, Perú, p. 23- 27, 2010.

\section{REFERENCIAS JURÍDICAS}

Acción de Inconstitucionalidad 26/2006. Recuperado de https://www.senado.gob.mx/comisiones/justicia/docs/Ministros/Zambrana/ Acccion26-2006.pdf

Código de Ética de las personas servidoras públicas del Gobierno Federal. (2019). https://www. gob.mx/cms/uploads/attachment/file/464527/ Co_digo_de_E_tica_SFP_2019_ok.pdf
Constitución Política de los Estados Unidos Mexicanos (05 de febrero de 1917).

Ley de Adquisiciones, Arrendamientos y Servicios del Sector Público. (04 de enero del 2000). Ciudad de México: Diario Oficial de la Federación. https://www.gob.mx/cms/ uploads/attachment/file/120266/Ley_de_Adquisiciones_Arrendamientos_y_Servicios_ del_Sector_P_blico.pdf

Ley de Asociaciones Público Privadas. (16 de enero del 2012). Ciudad de México: Diario Oficial de la Federación. http://www.diputados.gob. mx/LeyesBiblio/pdf/LAPP_150618.pdf

Ley de Obras Públicas y Servicios Relacionados con las Mismas. (04 de enero del 2000). Ciudad de México: Diario Oficial de la Federación. http://www.oas.org/juridico/spanish/ mesicic3_mex_anexo29.pdf

Ley Federal de Austeridad Republicana. (19 de noviembre de 2019). Ciudad de México: Diario Oficial de la Federación. http:// www.diputados.gob.mx/LeyesBiblio/pdf/ LFAR_191119.pdf

Ley Federal de Procedimiento Administrativo. (04 de agosto de 1994). Ciudad de México: Diario Oficial de la Federación. Recuperado de: http://www.diputados.gob.mx/LeyesBiblio/ pdf/112_180518.pdf

Ley General de Responsabilidades Administrativas. (18 de julio del 2016). Ciudad de México: Diario Oficial de la Federación. Recuperado de: http://www.imss.gob.mx/sites/all/ statics/pdf/leyes/4340.pdf

Lineamientos generales para propiciar la integridad de los servidores públicos e implementar acciones que favorezcan su comportamiento ético. (20 de agosto del 2015). Ciudad de México: Diario Oficial de la Federación. Recuperado de: http://www.dof.gob. $\mathrm{mx} /$ nota_detalle.php?codigo $=5450952 \& \mathrm{fec}$ ha $=02 / 09 / 2016$ 
Protocolo de Actuación en materia de Contrataciones Públicas, otorgamiento y prorroga de Licencias, Permisos, Autorizaciones y Concesiones. (20 de agosto del 2015). Ciudad de México: Diario Oficial de la Federación. Recuperado de: https://www.gob.mx/sfp/ documentos/protocolo-de-actuacion-enmateria-de-contrataciones-publicas-otorga- miento-y-prorroga-de-licencias-permisosautorizaciones-y-concesiones-97983

Reglas de Integridad para el ejercicio de la Función Pública. (20 de agosto del 2015). Ciudad de México: Diario Oficial de la Federación. Recuperado de: https://www.gob.mx/cms/ uploads/attachment/file/188655/ReglasdelntegridadParaEjercicioFuncionPublica.pdf 